\title{
DEPENDÊNCIA DA TAXA DE DISSIPAÇÃO VISCOSA COM A ESTABILIDADE ATMOSFÉRICA EM CONDIÇÕES MUITO ESTÁVEIS
}

\author{
Felipe D. Costa $^{1}$, Leandro L. Gonzales ${ }^{1}$, Franciano S. Puhales ${ }^{2}$, Giuliano Demarco ${ }^{2}$, \\ Luis G. N. Martins ${ }^{2}$, Otávio C. Acevedo ${ }^{3}$ \\ ${ }^{1}$ Universidade Federal do Pampa, Campus Alegrete, RS, Brasil \\ ${ }^{2}$ Universidade Federal de Santa Maria, Santa Maria, RS, Brasil \\ *fdenardin@gmail.com
}

\section{RESUMO}

A descrição prognóstica do escoamento turbulento na camada limite muito estável é um desafio para modelos numéricos, pois os mecanismos que mantém a atividade turbulenta são desconhecidos. Neste trabalho, são analisadas as implicações das hipóteses de Kolmogorov na parametrização da taxa de dissipação viscosa. Os resultados indicam que esta deve depender do regime de escoamento atmosférico.

\begin{abstract}
The prognostic description of the turbulent flow in the very stable boundary layer is still a challenge for the numerical models, as the mechanisms that maintain the turbulent activity are unknown. In this work, the implications of the Kolmogorov hypothesis in the parameterization of the viscous dissipation rate are analyzed. The results indicate that the viscous dissipation rate should depend on the atmospheric flow regime.
\end{abstract}

\section{INTRODUÇÃO}

A descrição prognóstica do escoamento turbulento na camada limite estável (CLE) tem sua descrição particularmente dificultada em noites com forte estratificação térmica. Nestes casos, a teoria prevê que a mistura turbulenta deve ser completamente suprimida pela estabilidade atmosférica, todavia, o número de Reynolds é varias ordens de grandeza maior que o de um escoamento laminar, caracterizando assim um escoamento turbulento. Entretanto, os mecanismos responsáveis pela manutenção da turbulência, nestas condições são desconhecidos.

A proposta deste trabalho é que um dos mecanismos responsáveis pela manutenção da atividade turbulenta, mesmo em condições, é a taxa de dissipação viscosa da turbulência. Esse termo geralmente é parametrizado em modelos de ordem 1.5 do tipo "E-l", utilizando uma parametrização advinda da lei de Kolmogorov (KOLMOGOROV; 1941). Porém, tal lei é valida somente para turbulência completamente desenvolvida, homogênea e isotrópica, condições que não são satisfeitas no regime muito estável da CLE. Geralmente, nestas condições é incluída uma constante ${ }^{c}$, na expressão para a taxa de dissipação que indica o grau de anisotropia do escoamento. Neste trabalho é analisada a influência dessa aproximação em diferentes formulações de turbulência. 


\section{MODELO}

Para condições de uma atmosfera idealizada, utilizando fechamento de turbulência de ordem 1.5, as equações que descrevem o escoamento atmosférico na CLE, podem ser escritas como:

$$
\begin{gathered}
\frac{\partial u}{\partial t}=f\left(v-v_{G}\right)-\frac{\partial\left(u_{*}^{2} \cos \psi\right)}{\partial z} \\
\frac{\partial v}{\partial t}=f\left(u_{G}-u\right)-\frac{\partial\left(u_{*}^{2} \sin \psi\right)}{\partial z} \\
\frac{\partial \theta}{\partial t}=-\frac{\partial u_{*} \theta_{*}}{\partial z} \\
\frac{\partial E}{\partial t}=S u_{*}^{2}-R i S u_{*}^{2}+\left[K_{E} \frac{\partial E}{\partial Z}\right]-c_{\varepsilon} \frac{(\alpha E)^{3 / 2}}{l_{m}} \\
\frac{\partial \theta_{g}}{\partial t}=\frac{1}{C_{g}}\left(I_{\downarrow}-\sigma \theta_{g}^{4}-H_{0}\right)-k_{m}\left(\theta_{g}-\theta_{m}\right)
\end{gathered}
$$

Os fluxos turbulentos de momentum são estimados através da velocidade de fricção, que está relacionada com a ECT por: $u_{*}^{2}=\alpha E$. O fluxo de energia na forma de calor é função da escala de temperatura, que é calculada utilizando teoria $\mathrm{K}\left({ }^{\theta_{*}}=K_{h}(\partial \theta / \partial z)\right.$ ). Os coeficientes de difusão são parametrizados como: $K_{m}=\sqrt{\alpha E} l_{m} ; K_{H}=K_{m} / \operatorname{Pr}$; $K_{E}=K_{m} / \sigma_{E}$, sendo $\sigma_{E}=2,5$ e $\operatorname{Pr}=1$.

A dependência do escoamento com a estabilidade atmosférica é representada através de três formulações de turbulência: 1) considera que a relação entre mistura turbulenta e a estabilidade é dada diretamente através dos termos da equação (4); 2 i) Admite que o comprimento de mistura varie com a estabilidade atmosférica, $l_{m}^{3}=\kappa z \varphi(R i)$, onde $\varphi(R i)$ é uma função de estabilidade dada por: $\varphi(R i)=[1+4.7 R i]^{-2}$ (LOUIS; 1979); 3) Considera que em condições muito estáveis, a escala de tempo característica dos processos turbulento nas CLE é muito pequena, logo, processos não turbulentos de grande escala (mas com escala inferior aos processos de mesoescala (submeso)), têm grande influência sobre o comportamento dos fluxos turbulentos. Logo, uma relação adimensional que leva em consideração a influência destes processos é utilizada na estimativa da constante $\alpha$, como: $\alpha=\left[4+(1+2,5 / L)^{1 / 3}\right]^{-1}$. Finalmente, a constante $\mathrm{c}_{\varepsilon}$ advém do fato de que a condição de homogeneidade e isotropia não é satisfeita na CLE muito limite estável. Os valores aqui utilizados foram $c_{\varepsilon}: 0,1$ (caso mais longe das condições de isotropia e homogeneidade), 0,2, $0,4,0,6,0,8$ e 1 (turbulência isotrópica e homogênea).

\section{RESULTADOS}

A figura 1 mostra a relação média entre a intensidade da turbulência dada por ${ }^{u_{*}} \mathrm{e}$ a estabilidade atmosférica dada por $R i$, utilizando diferentes valores para a constante que representa indica o grau de isotropia do escoamento turbulento na CLE. Para todas as formulações utilizadas à medida que ${ }^{c_{\varepsilon}}$ aumenta, o valor do número de Richardson mínimo necessário para que a turbulência seja contínua no escoamento diminui indicando que o valor 
de $c_{\varepsilon}$ deve apresentar dependência com a estabilidade, e o valor critico de $R i$ não deve ser constante no regime muito estável da CLE.

A figura 1 também mostra que no estado conectado, caracterizado pela presença de turbulência contínua, a consideração da hipótese de Kolmogorov leva aos melhores já que a dissipação elevada evita valores irreais de $\boldsymbol{u}_{*}$.
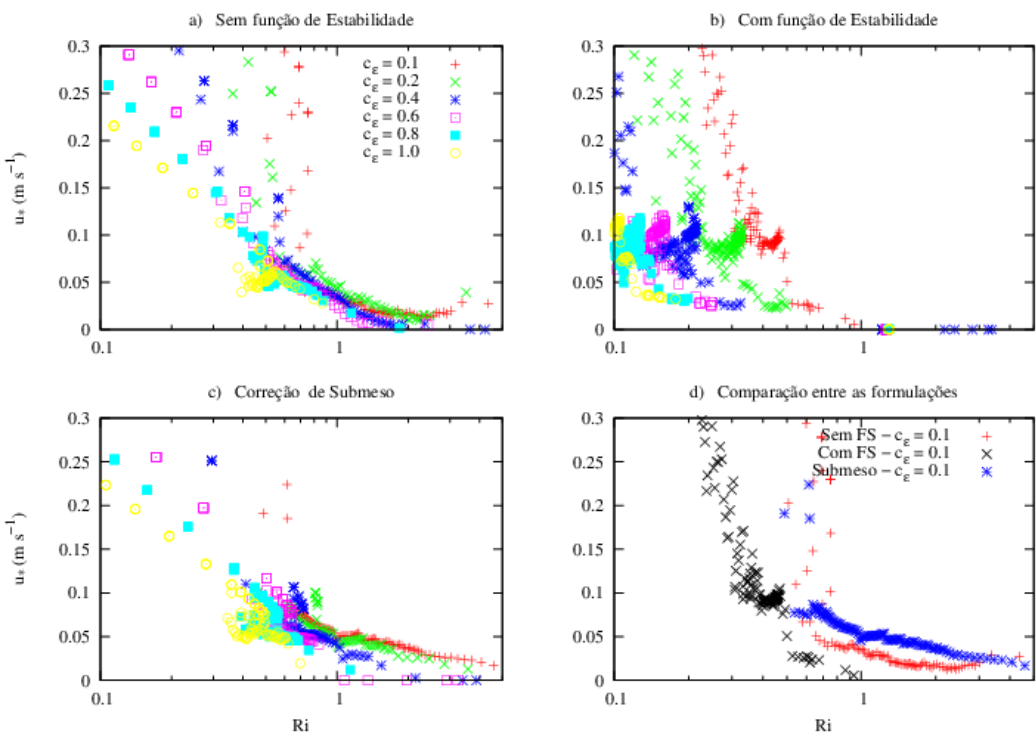

Figura 1: Dependência da intensidade turbulenta com a estabilidade atmosférica para diferentes valores de $c_{\varepsilon}$.

\section{CONCLUSÃO}

Os resultados indicam que a manutenção da atividade turbulenta, mesmo no regime muito estável da CLE, pode estar relacionada com taxa de dissipação viscosa de ECT, a qual deve ser drasticamente reduzida no estado desconectado, através de um fator adimensional que depende da estratificação térmica.

\section{REFERÊNCIAS BIBLIOGRÁFICAS}

ACEVEDO, O. C., COSTA, F. D., OLIVEIRA, P. E., PUHALES, F. S., DEGRAZIA, G. A., ROBERTI, D. R., 2013: The influence of submeso processes on stable boundary layer similarity relationships. J. of the Atmos. Sci., e-view, doi: 10.1175/JAS-D-13-0131.1.

LOUIS J.F., 1979: A parametric model of vertical fluxes in the atmosphere. Boundary-Layer Meteorol., 17, 187-202.

KOLMOGOROV A.N., 1941: Dissipation of energy in a locally isotropic turbulence. Doklady Akad. Nauk SSSR. 